PROCEEDINGS OF THE

AMERICAN MATHEMATICAL SOCIETY

Volume 132, Number 2, Pages 317-323

S 0002-9939(03)07027-8

Article electronically published on June 17, 2003

\title{
ON THE ERROR TERM IN AN ASYMPTOTIC FORMULA FOR THE SYMMETRIC SQUARE $L$-FUNCTION
}

\author{
YUK-KAM LAU
}

(Communicated by Wen-Ching Winnie Li)

Abstract. Recently $\mathrm{Wu}$ proved that for all primes $q$,

$$
\sum_{f} L\left(1, \operatorname{sym}^{2} f\right)=\frac{\pi^{4}}{432} q+O\left(q^{27 / 28} \log ^{B} q\right)
$$

where $f$ runs over all normalized newforms of weight 2 and level $q$. Here we show that $27 / 28$ can be replaced by $9 / 10$.

\section{INTRODUCTION}

Let $q$ be a prime and

$$
\Gamma_{0}(q)=\left\{\left(\begin{array}{ll}
a & b \\
c & d
\end{array}\right) \in S L_{2}(\mathbb{Z}): q \mid c\right\} .
$$

We denote by $S_{2}(q)$ the space of all holomorphic cusp forms for $\Gamma_{0}(q)$ of weight 2 . With respect to the inner product

$$
\langle f, g\rangle=\int_{\Gamma_{0}(q) \backslash \mathbf{H}} f(z) \overline{g(z)} d x d y,
$$

$S_{2}(q)$ is a finite-dimensional Hilbert space, and there is an orthogonal basis $\mathcal{B}_{2}(q)$ (which is the set of all normalized newforms in $S_{2}(q)$ ) such that

(i) each $f \in \mathcal{B}_{2}(q)$ is a common eigenvector of all Hecke operators $T_{n}$ with $(n, q)=1$, i.e. when $f \in \mathcal{B}_{2}(q)$ and $(n, q)=1$,

$$
T_{n} f=\lambda_{f}(n) f
$$

(ii) the Fourier expansion of $f \in \mathcal{B}_{2}(q)$ is

$$
f(z)=\sum_{n=1}^{\infty} \lambda_{f}(n) \sqrt{n} e(n z)
$$

where $e(\alpha)=e^{2 \pi i \alpha}, \lambda_{f}(n)$ is the eigenvalue in (i) if $(n, q)=1$ and $\lambda_{f}(n)^{2}=$ $l^{-1} \lambda_{f}(m)^{2}$ if $n=l m$ where $l$ is a power of $q$ and $(m, q)=1$ (see [3 (2.19) and (2.24)]).

Received by the editors September 17, 2002.

2000 Mathematics Subject Classification. Primary 11F67.

(C)2003 American Mathematical Society 
For the properties of $\lambda_{f}(n)$, it is known that they are all real and satisfy the Deligne bound $\left|\lambda_{f}(n)\right| \leq \tau(n)$. (Here and in the sequel $\tau(n)=\sum_{d \mid n} 1$ is the divisor function.) Moreover we have

$$
\lambda_{f}(m) \lambda_{f}(n)=\sum_{d \mid(m, n)} \epsilon_{q}(d) \lambda_{f}\left(\frac{m n}{d^{2}}\right)
$$

where $\epsilon_{q}$ is the principal character $\bmod q$. In particular, we see that $\lambda_{f}(1)=1$. Associated to each $f \in \mathcal{B}_{2}(q)$, we define the symmetric square $L$-function by

$$
L\left(s, \operatorname{sym}^{2} f\right)=\zeta_{q}(2 s) \sum_{n=1}^{\infty} \lambda_{f}\left(n^{2}\right) n^{-s} \quad \text { for } \Re e s>1,
$$

where $\zeta_{q}(s)=\prod_{p \nmid q}\left(1-p^{-s}\right)^{-1}$. This $L$-function extends to an entire function over $\mathbb{C}$ and it satisfies a functional equation; more precisely, let us write

$$
\Lambda\left(s, \operatorname{sym}^{2} f\right)=\left(\frac{q}{\pi^{3 / 2}}\right)^{s} \Gamma\left(\frac{s+1}{2}\right)^{2} \Gamma\left(\frac{s+2}{2}\right) L\left(s, \operatorname{sym}^{2} f\right) .
$$

Then we have $\Lambda\left(s, \operatorname{sym}^{2} f\right)=\Lambda\left(1-s, \operatorname{sym}^{2} f\right)$. Analogous to the Riemann zeta function, the values attained by $L\left(s, \operatorname{sym}^{2} f\right)$ in the critical strip are interesting. Particularly for $s=1$ and all large prime $q$, we have the asymptotic formula

$$
\sum_{f \in \mathcal{B}_{2}(q)} L\left(1, \operatorname{sym}^{2} f\right)=\frac{\pi^{4}}{432} q+O\left(q^{\alpha} \log ^{\beta} q\right)
$$

for some constants $0<\alpha<1$ and $\beta>0$. Here, we are concerned with the size of the error term. In [1, Akbary proved that $\alpha=45 / 46$ is admissible and recently Wu gave an improvement to $\alpha=27 / 28$ (see [5]). Our purpose is to show the refinement below.

Theorem. Let $q$ be a prime. There is an absolute constant $c>0$ such that

$$
\sum_{f \in \mathcal{B}_{2}(q)} L\left(1, \operatorname{sym}^{2} f\right)=\frac{\zeta(2)^{3}}{2 \pi^{2}} q+O\left(q^{9 / 10} \log ^{c} q\right) .
$$

(Note that $\zeta(2)^{3} /\left(2 \pi^{2}\right)=\pi^{4} / 432$.)

Remark. In decimal form we have $\frac{45}{46} \approx 0.978, \frac{27}{28} \approx 0.964$ and $\frac{9}{10}=0.9$.

\section{SOME PREPARATION}

Lemma 1. Let $A>1$ be any fixed constant and $q \ll y \ll q^{A}$ but $y \notin \mathbb{Z}$. We have

$$
L\left(1, \operatorname{sym}^{2} f\right)=\zeta_{q}(2) \sum_{n \leq y} \frac{\lambda_{f}\left(n^{2}\right)}{n}+O\left(q^{\epsilon}\left(y^{-1}+\left(\frac{q}{y}\right)^{2 / 7}\right)\right)
$$

where $\epsilon>0$ is an arbitrarily small constant and the implied constant in the O-term depends on $\epsilon$.

Proof. This follows from the truncated Perron's formula. Using the estimate

$$
\Gamma\left(\frac{s+1}{2}\right)^{2} \Gamma\left(\frac{s+2}{2}\right) \asymp|t|^{(3 \sigma+1) / 2} e^{-3 \pi|t| / 4}
$$

for $s=\sigma+i t$ where $\sigma \ll 1$ and $|t| \gg 1$, we can derive from the functional equation the convexity bound: for $0 \leq \sigma \leq 1$,

$$
L\left(\sigma+i t, \operatorname{sym}^{2} f\right) \ll\left(q|t|^{3 / 2}\right)^{1-\sigma+\epsilon} .
$$


By [2, Lemma 12.1], we see that for any $T \gg 1$,

$$
\begin{gathered}
\zeta_{q}(2) \sum_{n \leq y} \frac{\lambda_{f}\left(n^{2}\right)}{n} \\
(5)=\frac{\zeta_{q}(2)}{2 \pi i} \int_{\epsilon-i T}^{\epsilon+i T} \sum_{n=1}^{\infty} \frac{\lambda_{f}\left(n^{2}\right)}{n^{1+s}} \frac{y^{s}}{s} d s+O\left(y^{\epsilon} \sum_{n=1}^{\infty} \frac{\tau(n)^{2}}{n^{1+\epsilon}} \min \left(1,\left(T\left|\log \frac{y}{n}\right|\right)^{-1}\right)\right) .
\end{gathered}
$$

To evaluate the $O$-term, we split the summation over $n$ into three pieces: $n \leq y / 2$, $n \geq 3 y / 2$ and $y / 2<n<3 y / 2$. As $|\log (y / n)| \gg 1$ in the first two pieces, these two sums are $O\left(T^{-1} y^{\epsilon}\right)$. The third one is

$$
\ll y^{\epsilon} T^{-1} \sum_{\substack{y / 2<n<3 y / 2 \\|n-y| \geq 1}}|y-n|^{-1}+y^{-1+\epsilon} \ll y^{\epsilon}\left(T^{-1}+y^{-1}\right) .
$$

Thus the overall contribution is absorbed in the $O$-term in our lemma.

From (2), we can replace $\sum_{n=1}^{\infty} \lambda_{f}\left(n^{2}\right) n^{-(1+s)}$ in (5) by

$$
\zeta_{q}(2+2 s)^{-1} L\left(1+s, \operatorname{sym}^{2} f\right) \text {. }
$$

Then we apply the residue theorem to the rectangular contour with vertices at $\epsilon \pm i T$ and $-1 / 2+\epsilon \pm i T$. The integral in (5) equals a sum of two terms: the main term $L\left(1, \operatorname{sym}^{2} f\right)$ from the pole at $s=0$, and the remainder term which is

$$
\begin{aligned}
& \ll \int_{-1 / 2+\epsilon}^{\epsilon}\left|\frac{L\left(1+\alpha+i T, \mathrm{sym}^{2} f\right)}{\zeta_{q}(2+2 \alpha+i 2 T)}\right| \frac{y^{\alpha}}{T} d \alpha+y^{-1 / 2+\epsilon} \\
& \cdot \int_{-T}^{T}\left|\frac{L\left(1 / 2+\epsilon+i t, \mathrm{sym}^{2} f\right)}{\zeta_{q}(1+2 \epsilon+i 2 t)}\right| \frac{d t}{1+|t|} .
\end{aligned}
$$

Using the bound $\zeta(\sigma+i t)^{-1} \ll \log (1+|t|)$ for $\sigma \geq 1$ and $|t| \gg 1$, the two $O$-terms are $\ll(q T)^{\epsilon}\left(y^{-1 / 2} q^{1 / 2} T^{3 / 4}+T^{-1}\right)$. The proof is complete after setting $T=(y / q)^{2 / 7}$.

Our next task is to extend the admissible range in [5, Lemma 2]. To this end, we modify the mean square estimate result in [4. Corollary 1]. Suppose $M \leq q^{9}$ and $\left\{a_{n}\right\}_{1 \leq n \leq M}$ is a sequence of complex numbers. Then by taking $a_{n}=0$ for $M<n \leq q^{9}$, [4, Proposition 1] with $N=q^{9}$ gives

$$
\sum_{f \in \mathcal{B}_{2}(q)}\left|\sum_{n \leq M} a_{n} \rho_{f}(n)\right|^{2} \ll q^{9}(\log q)^{15} \sum_{n \leq M}\left|a_{n}\right|^{2}
$$

where $\rho_{f}(n)=\sum_{l m^{2}=n} \epsilon_{q}(m) \lambda_{f}\left(l^{2}\right)$. (Note that $\mathcal{B}_{2}(q)=S_{2}(q)^{*}$ in [4] for prime $q$.)

Lemma 2. Let $M \gg 1$ and suppose that $\{a(n)\}_{M<n \leq 2 M}$ satisfies

$$
a(n) \ll \frac{(\tau(n) \log n)^{A}}{n}
$$

for some constant $A>0$. There exists a constant $B=B(A) \geq 0$ such that

$$
\sum_{f \in \mathcal{B}_{2}(q)}\left|\sum_{M<n \leq 2 M} a(n) \lambda_{f}\left(n^{2}\right)\right|^{2} \ll \max \left(1, q^{9} M^{-1}\right) \log ^{B}(q M) .
$$

The implied constant depends on A. 
Proof. When $M \geq q^{9}$, it follows immediately from [4, Corollary 1] (by taking $N=M)$. Consider the case $M<q^{9}$. From [4, (16)], we have

$$
S:=\sum_{f \in \mathcal{B}_{2}(q)}\left|\sum_{M<n \leq 2 M} a(n) \lambda_{f}\left(n^{2}\right)\right|^{2}=\sum_{f \in \mathcal{B}_{2}(q)}\left|\sum_{l<2 M} a_{l} \rho_{f}(l)\right|^{2}
$$

where

$$
\begin{aligned}
a_{l} & =\sum_{\sqrt{M / l}<m \leq \sqrt{2 M / l}} \mu(m) \epsilon_{q}(m) a\left(l m^{2}\right) \\
& \ll \frac{(\tau(l) \log 2 l)^{A}}{l} \sum_{\sqrt{M / l}<m \leq \sqrt{2 M / l}} \frac{(\tau(m) \log 2 m)^{2 A}}{m^{2}} \\
& \ll(M l)^{-1 / 2}(\log M l)^{B}
\end{aligned}
$$

(see the proof of [4, Corollary 1] as well). $B$ denotes an unspecified positive constant depending on $A$ and its value may differ at each occurrence in the proof. By (6),

$$
S \ll q^{9}(\log q)^{15} \sum_{l<2 M}(M l)^{-1}(\log M l)^{B} \ll q^{9} M^{-1} \log ^{B}(q M) .
$$

Define for $1 \leq x<y$,

$$
\omega_{f}(x, y)=\sum_{x \leq n<y} \frac{\lambda_{f}\left(n^{2}\right)}{n} .
$$

Lemma 3. Let $x>0$ and $x<y \ll q^{A}$ for some constant $A>0$. Suppose $r \geq 1$ is a fixed integer satisfying $x^{r} \geq q^{9}$. Then there exists a constant $D=D(r)>0$ such that

$$
\sum_{f \in \mathcal{B}_{2}(q)} \omega_{f}(x, y)^{2 r} \ll(\log q)^{D}
$$

where the implied constant depends on $A$ and $r$.

Proof. Following the argument in the proof of [4, Lemma 4], one can show that

$$
\omega_{f}(x, y)^{r}=\sum_{x^{r} \leq m n<y^{r}} \lambda_{f}\left(m^{2}\right) \frac{c(m, n)}{m n}
$$

where $c(m, n)$ is independent of $f$ and $c(m, n)=0$ if $n$ is not of the form $n=d n_{1}$ where $d \mid m$ and $n_{1}$ is squarefull. Moreover, $|c(m, n)| \leq \tau(m n)^{\gamma}$ for some integer $\gamma=\gamma(r)>0$ depending on $r$. Then we write

$$
\omega_{f}(x, y)^{r}=\sum_{H=2^{k}} \sum_{\substack{x^{r} \leq m n<y^{r} \\ H \leq n<2 H}} \lambda_{f}\left(m^{2}\right) \frac{c(m, n)}{m n}
$$

where the first summation runs over all nonnegative integers $k$. Define

$$
c_{H}(m)=\sum_{\substack{H \leq n<2 H \\ x^{r} m^{-1} \leq n<y^{r} m^{-1}}} \frac{c(m, n)}{n} .
$$


Then, using $\sum_{\substack{n \leq z \\ \text { squarefull }}} \tau(n)^{\gamma} \ll z^{1 / 2}(\log z)^{2^{\gamma}}$, we have

$$
\begin{aligned}
c_{H}(m) & \ll \tau(m)^{\gamma} \sum_{d \mid m} \frac{1}{d} \sum_{\substack{H \leq d n<2 H \\
n \text { squarefull }}} \frac{\tau(n)^{\gamma}}{n} \\
& \ll \tau(m)^{\gamma}\left(\sum_{\substack{d \mid m \\
d>\sqrt{H}}} d^{-1}+\sum_{\substack{d \mid m \\
d<\sqrt{H}}} d^{-1} \sum_{\substack{H / d \leq n<2 H / d \\
n \text { squarefull }}} \frac{\tau(n)^{\gamma}}{n}\right) \\
& \ll H^{-1 / 2}(\tau(m)(\log m)(\log H))^{D} .
\end{aligned}
$$

Here we use $D$ to denote a positive constant (depending on $r$ ) which may assume different values at other places. Making use of (7) for $H \geq q$,

$$
\omega_{f}(x, y)^{r}=\sum_{H=2^{k}<q} \sum_{x^{r} /(2 H)<m \leq y^{r} / H} \lambda_{f}\left(m^{2}\right) \frac{c_{H}(m)}{m}+O\left(q^{-1 / 2} \log ^{D} q\right) .
$$

Squaring both sides and averaging over all $f \in \mathcal{B}_{2}(q)$ yields

$$
\begin{aligned}
& \sum_{f \in \mathcal{B}_{2}(q)} \omega_{f}(x, y)^{2 r} \\
\ll & \left(\sum_{H=2^{k}<q} H^{-1} \sum_{f}\left|\sum_{x^{r} /(2 H)<m \leq y^{r} / H} \lambda_{f}\left(m^{2}\right) \frac{c_{H}(m) \sqrt{H}}{m}\right|^{2}+1\right) \log ^{D} q
\end{aligned}
$$

as $\left(\sum_{i \in I} a_{i}\right)^{2} \ll|I| \sum_{i \in I} a_{i}^{2}$ and $\left|\mathcal{B}_{2}(q)\right| \ll q$. For each $H$, we split the range of the summation over $m$ into dyadic intervals $M<m \leq 2 M$ where $M \geq x^{r} /(2 H)$. It follows from Lemma 2 and (7) that

$$
\sum_{f}\left|\sum_{x^{r} /(2 H)<m \leq y^{r} / H} \lambda_{f}\left(m^{2}\right) \frac{c_{H}(m) \sqrt{H}}{m}\right|^{2} \ll \max \left(1, q^{9} x^{-r} H\right) \log ^{D} q .
$$

Inserting it into (8), we conclude that

$$
\sum_{f \in \mathcal{B}_{2}(q)} \omega_{f}(x, y)^{2 r} \ll \log ^{D} q \sum_{H=2^{k}<q} \max \left(H^{-1}, q^{9} x^{-r}\right),
$$

and our result follows in view of the condition $x^{r} \geq q^{9}$.

\section{Proof of the Theorem}

Define for $f \in \mathcal{B}_{2}(q), w_{f}=4 \pi\langle f, f\rangle$, which is a positive real number. We have from [3, Lemma 2.5] that $w_{f}=\left(2 \pi^{2}\right)^{-1} q L\left(1, \mathrm{sym}^{2} f\right)$ and from [3, Corollary 2.2] (with $\left.\tau_{3}((m, n)) \leq \tau((m, n))^{2} \leq \tau(m) \tau(n)\right)$ that

$$
\sum_{f \in \mathcal{B}_{2}(q)} w_{f}^{-1} \lambda_{f}\left(m^{2}\right) \lambda_{f}\left(n^{2}\right)=\delta(m, n)+O\left(q^{-1}(m n)^{1 / 2}(\tau(m) \tau(n))^{2} \log 2 m n\right)
$$

for $\min (m, n)<q$, where $\delta(\cdot, \cdot)$ is the Kronecker delta. (Note that $w_{f}^{-1}=\omega_{f}$ in [4].) In particular, $\sum_{f} w_{f}^{-1} \ll 1$ as $\lambda_{f}(1)=1$.

We split the sum over $n$ in Lemma 1 into two subsums $\sum_{n \leq x}+\sum_{x<n \leq y}$ where $1<x<q<y$. (Our choice will be $x=q^{9 / 10}$ and $y=q^{173 / 110}$.) Squaring the 
formula in Lemma \together with the bound $L\left(1, \operatorname{sym}^{2} f\right) \ll \log ^{3} q$ (from [4 (18)]), we deduce that

$$
\begin{aligned}
\sum_{f \in \mathcal{B}_{2}(q)} L\left(1, \operatorname{sym}^{2} f\right) & =\frac{q}{2 \pi^{2}} \sum_{f \in \mathcal{B}_{2}(q)} w_{f}^{-1} L\left(1, \operatorname{sym}^{2} f\right)^{2} \\
& =\frac{q}{2 \pi^{2}} \zeta_{q}(2)\left(S_{1}+2 S_{2}+S_{3}\right)+O\left(q^{\epsilon}\left(y^{-1}+\left(\frac{q}{y}\right)^{2 / 7}\right)\right)
\end{aligned}
$$

where

$$
\begin{aligned}
S_{1} & =\sum_{f} w_{f}^{-1}\left(\sum_{n \leq x} \frac{\lambda_{f}\left(n^{2}\right)}{n}\right)^{2}, \\
S_{2} & =\sum_{f} w_{f}^{-1}\left(\sum_{n \leq x} \frac{\lambda_{f}\left(n^{2}\right)}{n}\right)\left(\sum_{x<n \leq y} \frac{\lambda_{f}\left(n^{2}\right)}{n}\right), \\
S_{3} & =\sum_{f} w_{f}^{-1}\left(\sum_{x<n \leq y} \frac{\lambda_{f}\left(n^{2}\right)}{n}\right)^{2} .
\end{aligned}
$$

It follows from the bound $w_{f}^{-1} \ll q^{-1} \log q$ (see [4 (20)]) and Lemma 3 that if $x^{r} \geq q^{9}$,

$$
\begin{aligned}
S_{3} & \ll \frac{\log q}{q} \sum_{f} \omega_{f}(x, y)^{2} \ll\left(\sum_{f} \omega_{f}(x, y)^{2 r}\right)^{1 / r}\left|\mathcal{B}_{2}(q)\right|^{1-1 / r} q^{-1} \log q \\
& \ll q^{-1 / r} \log ^{c_{11}} q .
\end{aligned}
$$

Throughout $c_{i}, i=1,2, \cdots$, denote unspecified positive constants. Using (9), we obtain that for $x<q$,

$$
\begin{aligned}
S_{1} & =\sum_{n \leq x} n^{-2}+O\left(q^{-1} \sum_{m, n \leq x}(m n)^{-1 / 2} \tau(m)^{2} \tau(n)^{2} \log 2 m n\right) \\
& =\zeta(2)+O\left(x^{-1}+q^{-1} x \log ^{c_{11}} q\right) .
\end{aligned}
$$

To treat $S_{2}$, we split it into two parts: let $z=q x^{-1}$,

$$
\begin{aligned}
S_{2} & =\sum_{f} w_{f}^{-1} \sum_{n \leq z} \frac{\lambda_{f}\left(n^{2}\right)}{n} \sum_{x<n \leq y} \frac{\lambda_{f}\left(n^{2}\right)}{n}+\sum_{f} w_{f}^{-1} \sum_{z<n \leq x} \frac{\lambda_{f}\left(n^{2}\right)}{n} \sum_{x<n \leq y} \frac{\lambda_{f}\left(n^{2}\right)}{n} \\
& =S_{21}+S_{22}, \text { say. }
\end{aligned}
$$

By (9), we have, provided that $z \leq x$ (or equivalently $x \geq q^{1 / 2}$ ),

$$
S_{21} \ll q^{-1}\left(\log ^{c_{11}} q\right) \sum_{m \leq z} \sum_{n \leq y} \tau(m)^{2} \tau(n)^{2}(m n)^{-1 / 2} \ll \sqrt{\frac{y}{q x}} \log ^{c_{11}} q .
$$

Applying the argument in (12), we get that

$$
\sum_{f} w_{f}^{-1}\left(\sum_{z<n \leq x} \frac{\lambda_{f}\left(n^{2}\right)}{n}\right)^{2} \ll z^{-1}+q^{-1} x \log ^{c_{11}} q \ll q^{-1} x \log ^{c_{11}} q .
$$


By $a b \ll|a|^{2}+|b|^{2}$ and (11), we have $S_{22} \ll\left(q^{-1 / r}+q^{-1} x\right) \log ^{c_{11}} q$. Hence, by (13),

$$
S_{2} \ll\left(q^{-1 / r}+q^{-1} x+\left(\frac{y}{q x}\right)^{1 / 2}\right) \log ^{c_{11}} q .
$$

Putting this estimate, (11) and (12) into (10), we infer that as $\zeta_{q}(2)=\zeta(2)+O\left(q^{-2}\right)$,

$$
\begin{aligned}
\sum_{f \in \mathcal{B}_{2}(q)} L\left(1, \operatorname{sym}^{2} f\right)=\frac{\zeta(2)^{3}}{2 \pi^{2}} q+q O\left(\left(q^{-1 / r}+\right.\right. & \left.q^{-1} x\right) \log ^{c_{11}} q \\
& \left.+q^{\epsilon}\left(x^{-1}+\left(\frac{y}{q x}\right)^{1 / 2}+\left(\frac{q}{y}\right)^{2 / 7}\right)\right) .
\end{aligned}
$$

Subject to the condition $x^{r} \geq q^{9}$, we take $x=q^{9 / r}$ and select $r=10, x=q^{9 / 10}$ and $y=q^{173 / 110}$ by equating $q^{-1 / r}=q^{-1} x$. This ends the proof.

\section{REFERENCES}

[1] A. Akbary, Average values of symmetric square L-functions at $\Re$ es $=2, \mathrm{C} . \mathrm{R}$. Math. Rep. Acad. Sci. Canada 22 (2000), 97-104. MR 2001h:11067

[2] A. Ivić, "The Riemann Zeta-Function," Wiley, New York, 1985. MR 87d:11062

[3] H. Iwaniec, W. Luo and P. Sarnak, Low lying zeros of families of L-functions, Inst. Hautes Études Sci. Publ. Math. 91 (2000), 55-131. MR 2002h:11081

[4] E. Kowalski and P. Michel, The analytic rank of $J_{0}(q)$ and zeros of automorphic L-functions, Duke Math. J. 100 (1999), 503-542. MR 2001b:11060

[5] J. Wu, Average values of symmetric square L-functions at the edge of the critical strip, Proc. Amer. Math. Soc. 131 (2003), 1063-1070.

Department of Mathematics, The University of Hong Kong, Pokfulam Road, Hong KONG

E-mail address: yklau@maths.hku.hk 